\title{
Editorial
}

\section{Distinguished celebration for Professor Georg H. Miley by the University of Illinois, Urbana, Illinois, USA}

For fifty years, research work of George Miley at the University of Illinois, a special Fest-Conference was organized collecting a large number of collaborators, associates, and former students on 14, 15 April 2011 at Urbana, Illinois. His very intensive involvement with publication of journals, conference proceedings, and books includes his very successful activity as Editor-in-Chief of Laser and Particle Beams from 1992 to 2001. This Journal dedicated a special address (Hora, 1993) at Miley's $60^{\text {th }}$ birthday (Fig. 1) with a summary of his achievements in science and technology. This is a special opportunity to congratulate George Miley with great thanks from all colleagues involved with Laser and Particle Beams.

The following may be an overview of Miley's enormous achievements on lasers, fusion energy, and nuclear technology including an integrated view about all his related activities. This may be underlined in view of the special present success on laser driven fusion (Glenzer et al., 2011) where Miley contributed in very many ways. This refers to his success with particle beam excited laser, an obvious vision from the very beginning of the laser to use the particles of a fission reactor. In order to pioneer this Miley had a chance to use the experiments with relativistic electron beams at Cornell University, aimed for another fusion direction by Norman Rostocker and Hans Fleischmann. Electron beam pumping of uniform semiconductors for laser emission experiments were reported before by Hurwitz et al. (1964) where the electron beam flux threshold and the necessary sample geometry was identical with the predicted calculations published at a colloquium at the Siemens Research Laboratory in Munich in April 1964 and distributed by Benoist de la Guillome at the semiconductor conference in Paris, June 1964 (see Hora, 2005). In contrast to this, the $\mathrm{CO}_{2}$ laser was from the beginning based on gas discharges, but to apply electron beam excitation was first done by Miley and subsequently led to his pioneering excitation of excimer lasers by e-beam irradiation of noble gases mixed with fluorine (Miley, 1972). This gave him the way to establish the activities with his TRIGA research reactor in his
Department of Nuclear Engineering at the University of Illinois to reach the first laser pumped with the neutrons from fission (DeYong et al., 1976) making him the father of the Nuclear Pumped Laser.

In view of the next options of laser drivers for the basic solution for mankind for solving the energy problem by nuclear fusion, the availability of the best lasers for driving will be a key question. At least the potential to expect lasers with pulses in the $50 \mathrm{MJ}$ range of duration not very much longer than nanoseconds was elaborated (Miley, 1977).

When Sir Isaac Newton was asked how he was able to discover key physics and mathematics behind our modern world, he replied that he had the opportunity to "stand on the shoulders of giants." This permits a comparison with George Miley. His remarkable achievements in science and engineering have moved on from existing technology to provide new centenary visions and in the process he has inspired numerous students to begin on this path. The diversification of groundbreaking discoveries by Miley has involved starkly different directions. However, there has always been the common thread of nuclear energy involved. Even his recent involvement with fuel cells for automobiles has a nuclear origin in that nuclear power plants are viewed as the energy source of choice for production of hydrogen needed for full scale operation of the future "hydrogen economy." His work is generally characterized as attacking an important application or societal need, e.g., his pioneering work on the plasma physics of neutron sources using inertial electrostatic confinement fusion has most important implications for neutron activation analysis used in Homeland Security detection systems. Miley's discoveries related to driven subcritical operation for improved safety in nuclear fission reactors (Hora et al., 2003) seems even more important now in light of the nuclear reactor damage at the Fukushima Daiichi nuclear power plant causing radioactive leakage following the earthquake-tsunami in Japan. The monstrous mistakes of engineers to build reactors in the tsunami range and not to use sufficient back-up cooling systems is nearly 


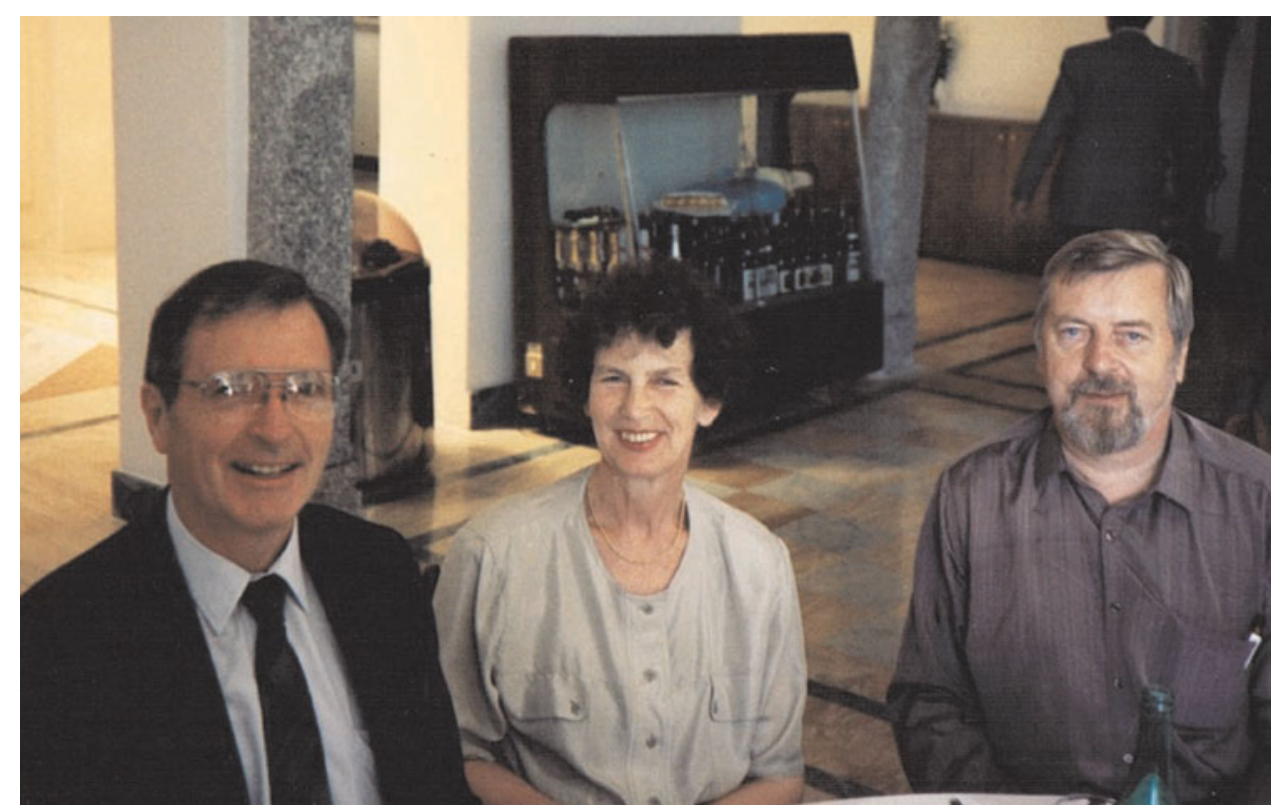

Fig. 1. (Color online) Professor George Miley (from left) with Rose Hora and Heinrich Hora whose $60^{\text {th }}$ birthday was celebrated on 1 July 1991 at Paradiso/Lago da Lugano, Switzerland.

unforgiveable while the shut-down of the reactors by its advanced safety systems worked perfectly. One of these systems is Miley and Hora's (1994) design.

Also noteworthy is his editorial work as editor of several major professional journals, his most successful and appreciated teaching and mentoring students, and his splendid organizing ability. An example of the later is the influential Laser-Plasmas Interaction Workshop series he coordinated for many years at the Naval Postgraduate School, Monterey, California. This workshop later became the current major international meeting series on inertial fusion science and applications of inertial confinement fusion. His effort as an Executive on the Board of Directors of the Society to Advance Fusion Energy spearheaded their successful campaign to promote a large increase in the budget for nuclear fusion research during the Presidency of Jimmy Carter.

In 1970, he began working out the first details for the nuclear fusion cross-sections of $\mathrm{p}^{\mathrm{B}} \mathrm{B}^{11}$ in the often cited crosssection book, Fusion Cross Sections and Reactivities. No neutrons are directly produced in this reaction, suggesting the possibility of a nuclear fusion reactor that would produce, on a per unit energy basis, less radioactivity in the entire process, including waste products, than from burning coal. In his profound book Miley (1976) elaborates the case for advanced fuel fusion energy well. Indeed, his fusion research has continuously been directed toward low neutron emission nuclear energy production, corresponding to the dream of a "golden age" where the ten million times more efficient nuclear energy replaces most of the chemical energy e.g., from fossil sources, with extremely clean and low cost power. The challenge mankind faces in getting to this goal is reflected in Miley's (1996) view of "Patience and Optimism," which was the title of his lecture when receiving the Edward Teller Medal in 1995.

The difficulty in plasma physics involved in fusion power was so well expressed by Edward Teller (2001) when referring to the situation in 1952. Teller summarized the problem in the following way: "Research on controlled fusion means dealing with the hydrodynamics of a plasma. I had a thorough respect for the fearsome nature of hydrodynamics, where every little volume does its own thing. Plasma does not consist of molecules, like a gas, but of ions - heavy slow moving positive ions - and light fast moving electrons. Those, in turn, create and are coupled with electric and magnetic fields. For each little volume of plasma, several questions have to be answered: How many positive ions? How many electrons? How fast does each move on the average? What is the electric force, and what is the magnetic force acting on them?

Mathematicians can predict the flow of matter as the volumes involved move in an orderly way. But even hydrodynamics of air was (and to some extend is - see weather forecasts) beyond the grasp of mathematics. Theoreticians of the nineteenth century proved that flying was impossible! In the twentieth century, they retreated to the statement that flying is impossible unless the air flow is confused and disordered (turbulent). Hydrodynamics as a science remains uncharted water.

The same complications occur in planning a thermonuclear explosion. But an explosion occurs in a so short time that many of the complicated phenomena have no chance to develop. Even so, it took a decade from Fermi's first suggestion of a thermonuclear reaction to the point (which occurred after the first full-scale demonstration of 
fusion) that the theoretical calculations of the explosions were reasonably complete. I had no doubt that demonstrating controlled fusion would be even more difficult."

This problem with plasma physics and hydrodynamics as related to controlled fusion power is still not fully solved some 60 years after Teller's comment, though much has been learned. Only research with great depth and breadth can lead us forward, as suggested by May (1972) and Haldane and May (2011) who ingeniously employed approaches used in theoretical physics to master complex systems mentioned by Teller. Thus, Lord May proposed solutions based on asking how "will a large complex system be stable," and began applying this approach to population systems in zoology, and finally to "systemic risk in banking systems" for analyzing the financial crisis. In similar ways, George Miley has approached problems by a combination of theory, computations, and experiments saying "I cannot be categorized as either a pure theorist or pure experimentalist. My guiding light came from Enrico Fermi's ability to analyze complex physics with simple elegant treatments that avoid excessive math but always supplied just enough to solve the problem at hand."

Miley has frequently stepped into the forefront of new explorations. Indeed, Miley was aware in advance of the crucial disclosure in March 1989 by Stephen Jones that led to the decision for a public announcement of cold fusion by Martin Fleischmann and Pons (1989). It is a tragedy how the subsequent confusion by claims, disclaims, and some untruths has poisoned this whole development. This turned many people, including most of the physics community, against cold fusion. Characteristically, Miley has done his best to keep an open mind and to dig down to the truth with his own independent research and also, as editor of several journals, sending cold fusion papers on to open minded reviewers (most physics journal editors have rejected such papers outright without review). His experimental work is very much different from most scientists who still work in cold fusion. He discovered the low energy nuclear reactions (LENRs) that produce nuclei over the whole range of elements detected using very sensitive analytic equipment in the Frederick Seitz laboratory at the University of Illinois (Miley et al., 2009). Miley's measurements show a remarkable small local maximum within the large scale minimum of the element distribution near the nucleon number $\mathrm{A}=$ 154. In comparison, such a local maximum occurs in the fission of excited Uranium at $\mathrm{A}=119$. This comparison confirms both to be a Maruhn-Greiner effect (Hora \& Miley, 2007) and provides a solid proof of the phenomenon of LENR, thus opening important directions for further exploration.

Another most intriguing discovery related to the LENR work is Miley's SQUID and superconducting measurement (Lipson et al., 2005) of the generation of clusters with more than 100 deuterons imbedded in the volume of one of the void defects (Schottky defect) created in palladium This is parallel to the later measurements of ultrahigh densities of clusters by Leif Holmlid (2009) in Gothenburg, Sweden where anomalously low laser intensity emissions of electrons and nuclear reactions have been detected (Badei et al., 2010). Both effects have been explained in a theory involving a Bose-Einstein like condensation. Experiments were performed to use the ultrahigh density in these clusters of inverted Rydberg states for having high density deuterium targets for laser fusion (Yang et al., 2011).

To round up this review, Miley's pioneering work for neutron-lean fusion reactions as that of $\mathrm{p}-\mathrm{B}^{11}$ may lead to a solution using petawatt-picsecond laser pulses to ignite a fusion flame in uncompressed solid density fuel. This is based on nonlinear force acceleration of plasma blocks where the accelerations are 100,000 times higher than by comparable thermal-pressure acceleration by lasers as predicted in 1978 and first measured by Sauerbrey in 1996, (see Fig. 10.18b of Hora, 1981; See Fig. 1 of Hora et al., 2007a; Hora, 2009). Miley had a key function to sort out the different options of volume ignition with spherical compression and thermal ignition as in the next solution (Miley et al., 2005) with NIF at Livermore (Glenzer et al., 2011) versus alternatives as fast ignition where the very fast interaction in the picosecond range may overcome the complications of complex systems as mentioned by Teller (2001) or Lord May (1972). The solution with side-on ignition of p-B ${ }^{11}$ (Hora et al., 2010) was described in an interview with the Royal Chemical Society of Chemistry in London by one of the leaders at the NIF experiment at Livermore, Steve Haan, that this "has the potential to be the best route to fusion energy" (Li, 2010). For the future steps, it may be interesting to consider Miley's electron beam excited $\mathrm{KrF}$ lasers as used in the large NIKE laser at the Naval Research Laboratory (Obenschain et al., 2006) with short pulse techniques (Földes and Szatmari, 2008).

Heinrich Hora University of New South Wales

\section{REFERENCES}

BAdiei, S., Andersson, P.U. \& Holmlid, L. (2010). Laser driven nuclear fusion D-D in ultradense deuterium: $\mathrm{MeV}$ particles formed without ignition. Laser Part. Beams 28, 313-317.

Deyong, R.J., Wells, W.E., Miley, G.H. \& Verdeyen, J.D. (1976). Direct nuclear pumping of a Ne- $\mathrm{N}_{2}$ laser. Appl. Phys. Lett. 28, 519-921.

Földes, I.B. \& Szatmari, S. (2008). On the use of KrF lasers for fast ignition. Laser Part. Beams 26, 575-582.

Fleischmann, M. \& Pons, S. (1989). Electrochemical induced nuclear fusion of deuterium. J. Electroan. Chem. 261, 301-308.

Glenzer, S.H., Moses, E.I., et al. (2011). Demonstration of ignition radiation temperatures in indirect-drive inertial confinement fusion hohlraums. Phys. Rev. Lett. 106, 085004/1-5.

Haldrane, A.G. \& May, R.M. (2011). Systemic risks in banking exosystems. Nature 469, 352-355. 
Holmlid, L., Hora, H., Miley, G.H. \& Yang, X. (2009). Ultrahigh density deuterium of Ryberg clusters for inertial confinement fusion targets. Laser Part. Beams 27, 529-532.

Hora, H. (2005). Contributions on Laser Driven Inertial Confinement Fusion. Am. J. Appl. Sci. 2, 1085-1094.

Hora, H. \& Miley, G.H. (2007). Maruhn-Greiner maximum from uranium fission for confirmation of low energy nuclear reactions LENR via a compound nucleus with double layer magic numbers. J. Fusion Energy 26, 349-354, 357.

Hora, H. \& Miley, G.H. (2007). Maruhn-Greiner maximum from uranium fission for confirmation of low energy nuclear reactions LENR via a compound nucleus with double layer magic numbers. J. Fusion Ener. 26, 349-356.

Hora, H. (1981). Physics of Laser Driven Plasmas, New York: Wiley-Interscience.

Hora, H. (1993). Dr. George H. Miley, Editorial. Laser Part. Beams, 11, 463-466.

Hora, H. (2009). Laser fusion with nonlinear force driven plasma blocks: Thresholds and dielectric effects. Laser Part. Beams 27, 207-222.

Hora, H., Badziak, J., Read, M.N., Li, Yu-Tong, Liang, Tian-Jiao, Liu Hong, Sheng Zheng-Ming, Zhang, Jie, Osman, F., Miley, G.H., Zhang, Weiyan, He, Xianto, Peng, Hanscheng, Glowacz, S., Jablonski, S., Wolowski, J., Skladanowski, Z., JungwiRth, K., Rohlena, K. \& Ullschmied, J. (2007a). Fast ignition by laser driven particle beams of very high intensity. Phys. Plasmas 14, 072701/1-7.

Hora, H., Miley, G.H., Ghornanneviss, M., Malekynia, B., Azizi, N. \& HE, X. (2010). Fusion energy without radioactivity: Laser ignition of solid density hydrogen-boron (11) fuel. Energy Environ. Sci. 3, 479-486.

Hora, H., Prelas, M.A. \& Miley, G.H. (2003). Nuclear Fission Reactors at Subcritical Operation. German Patent (DaimlerBenz) No. 4327920 C2 (granted 13.3.2003).

Hurwitz, C.G. \& Keyes, R.J. (1964). Electron beam pumped GaAs laser. Appl. Phys. Lett. 5, 139-141.

Li, Yuand (2010). Nuclear power without radioactivity. In Highlights in Chemical Technology. London: Royal Soc. Chem. 7, 1-2.
Lipson, A, Heuser, B.J., Castanov, C., Miley, G., Lyakov, B. \& Mitin, A. (2005). Transport and magnetic anomalies blow 70 $\mathrm{K}$ in a hydrogen cycled Pd foil with a thermally grown oxide. Phys. Rev. B72, 212507/1-6.

MaY, R.M. (1972). Will large complex systems be stable? Nature 238, 413-414.

Miley, G.H. (1972). Krypoton fluoride laser. Trans. Am. Nucl. Soc. 15, 2-6.

Miley, G.H. \& Hora, H. (1994). Pneumatic Safety Equipment to prevent the overheating of nuclear reactors, USA Patent 5,319,688.

Miley, G.H. (1976). Fusion Energy Conversion. Hinsdale, IL: American Nuclear Society.

Mirey, G.H. (1977). Direct nuclear power pumped lasers — Status and potential applications. In Laser Interaction and Related Plasma Phenomena. (Schwarz, H. \& Hora, H., Eds.), Vol. 4A, 181-228. New York: Plenum Press.

Miley, G.H. (1996). Patience and optimism. In Laser Interaction and Related Plasma Phenomena (Nakai, S. \& Miley, G.H., Eds.), Vol, 2, p. 1334-1352. Woodbury, NY: American Institute of Physics.

Miley, G.H., Hora, H., Osman, F., Evans, P. \& Toups, P. (2005). Single event laser fusion using ns-MJ laser pulses. Laser Part. Beams 23, 453-460.

Miley, G.H., Hora, H., Philberth, K, Lipson, A. \& Shrestha, P.L. (2009). Radiochemical comparisons on low energy nuclear reactions and uranium. In Low-Energy Nuclear Reactions and New Energy Technologies Source Book (Jan, Marwan and Steven B., Krivit, Eds.), Vol. 2, p. 235-252. Washington, DC: American Chemical Society/Oxford University Press.

Obenschain, S.P., Colombant, D.G., Schmitt, A.J., Sethian, J.D. \& Mcgroch, M.W. (2006). Pathway to a lower cost high repetition rate ignition facility. Phys. Plasmas 13, 056320.

Teller, E. (2001). Memoirs. Cambridge, MA: Perseus Publishing Perseus Publishing.

YAnG, X., Miley, G.H., Flippo, K.A. \& Hora, H. (2011). Energy enhancement for deuteron beam fast ignition of a pre-compressed inertial confinement fusion (ICF) target. Phys. Plasmas 18, $032703 / 1-7$. 